\title{
The Conversion Method from SVG Graphics for Substation to CIM/G Graphics for Intelligent Dispatching
}

\author{
Hao Ren \\ China Electric Power Research Institute \\ Nanjing, China \\ renhao@epri.sgcc.com.cn
}

\author{
Xiaoqing Ma \\ Nari Technology Development Limited Company \\ Nanjing, China \\ maxiaoqing@sgepri.sgcc.com.cn
}

\begin{abstract}
Based on the research of SVG and CIM/G vector graphics standards, through the analysis and comparison of the graphics files of SVG for substation and CIM/G for intelligent dispatching, the difference and correspondence relationship of basic and power graphics elements are found out. Adopting modular design ideas and cross platform technology, using dom $4 \mathrm{j}$ of Java xml parser to parse the element definition and each power graphics layer of SVG file for substation, hierarchical and classified metafile set of CIM/G format, graphics files of CIM/G format and graphics mapping point tables are generated, according to the mode of element type and state fusion, and graphics element mapping relationship. Proved by engineering practice, the method realizes the seamless connection from the monitoring graphics for substation to the intelligent dispatching system, and the automatic correspondence of the graphics signal points, which can shorten the time of substation graphics standardization operation greatly and reduce engineering pressure on manual point.
\end{abstract}

Keywords-SVG;CIM/G;DOM4J;Vector Conversion;Seamless Docking

\section{INTRODUCTION}

In order to support the operation requirements of the big run and the integration of dispatching and control, National Electric Power Dispatching Center points out the construction principles of substation system, of which refers to alarm direct transferring, remote browsing, data optimization and authentication security. It is pointed out that the substation system should have the function of remote browsing in the mode of big run obviously. Through the means of remote browsing to realize the substation panoramic information monitoring, dispatching monitor attendant or maintenance personnel needs to check the operation information of substation in detail, and can access to the motoring graphics and real-time graphics data at any time.

Because of the heterogeneous graphics format supported by the dispatching and substation system, the D5000 system of intelligent dispatching platform uses CIM/G standard as the file format for graphics subsystem, but the mainstream domestic monitoring system of substation generally supports the graphics file of SVG format. So, the dispatching and substation can't carry out graphics interaction and the function of conversion from SVG to CIM/G format needs to be achieved, which realize the unification of graphic format of the master and substation, and remove graphics heterogeneous barriers for the dispatching master browsing the monitoring graphics remotely.

\section{TECHNICAL BASIS}

\section{A. Substation Graphics Exchange Format Based on SVG}

$\mathrm{SVG}$ is a markup language for describing 2D graphics based on XML, the full name of which is scalable vector graphics. SVG graphics includes three parts of vector graphics, bitmap image and text. Graphics includes basic graphics and path. Although path can express all the graphics, six basic graphics of straight line, circle, ellipse, rectangle, polygon and dot are still defined in the SVG standard for painting convenience. In the aspect of image processing, two special effects of mask and filter are defined. In the aspect of text, the application features of arrangement by route, typography and embedded font are defined.

The SVG graphics exchange format for substation expands the information of electrical model elements and private attribute information based on standard SVG element information. The purpose is to realize the graphics exchange between different systems, different factories and power enterprises. This standard includes system graphics, bay graphics, wiring graphics of the power plants and stations, power flow graphics and so on. Its graphics file includes file header, the definition of element and style, power equipment component layer, static text layer, measurement layer, connection layer and so on. The relation of SVG graphics and neighborhood data uses metadata to describe. Metadata is a kind of data of describing data, mainly to describe some properties of data information [1] [2]. 


\section{B. CIM/G Graphics Description Specification for Power System}

The graphics description specification for power system is a kind of plain text language based on the markup of XML standard and compatible with basic graphics format of SVG, the name of which is $G$ language for short. $G$ language as special graphics format for power system is a new graphics description language applied in power system, which develops from the SVG graphics exchange format of the public information platform based on CIM/XML, and aims to the problem that the public graphics exchange format based on SVG cannot express the concept of power system and model integration directly.

The basic graphics element of $\mathrm{G}$ language inherits graphics description features and syntax rules of SVG, adopting multilevel reference mode of power equipment graphics object - > bay template - > power equipment element, and expressing and accessing power equipment information succinctly and effectively. The constituent elements of $\mathrm{G}$ language can be divided into basic graphics element and grid graphics element based on its type. The basic graphics element is used to draw graphics elements on the target canvas, which includes some standard shapes such as rectangle, circle, ellipse, straight line, broken line and polygon. The grid graphics element is used to express power equipment, divided into three categories including element, bay template and other mode [3].

\section{XML Parser DOM4J}

DOM4J is a XML parsing package for open source produced by dom4j.org, and is an easy-to-use and open source library for XML, XPath and XSLT. It is applied in java platform, adopting java framework and supporting DOM, SAX and JAXP fully. At the same time, DOM4J is a very good XML API for java, with some features of excellent performance, powerful function and easy to use.

The main interfaces of DOM4J are defined in the package of org.dom $4 \mathrm{j}$. Among them, Node defines the polymorphic behavior for all XML nodes in Dom4j. Branch defines a communal behavior for the node that can contain child nodes like Element and Document of XML. CharacterData is a markup interface, used to identify the node based on character. The rest of the interface such as DocumentType defines the DOCTYPE statement, Entity defines XML Entity, Attribute defines the property of XML, and ProcessingInstruction defines preprocessing instruction of XML [4].

\section{StRUCTURE ANALYSIS OF GRAPHICS FILE}

\section{A. File Structure of SVG Format}

The SVG format files for describing the substation monitoring graphics consist of two parts from the overall architecture. The first part is the definition of element and style, and the second part is the implementation of power graphics. The first part is contained within the <defs > element, just to define the elements referenced, not to draw and render any actual elements. The second part classifies the nodes of the various device types in the $\mathrm{G}$ group according to the power equipment type. Other elements not having specific equipment type are unified into Other_Layer. The <use> element can only realize the drawing and rendering of the power element. The file structure of SVG format is as shown in figure 1.

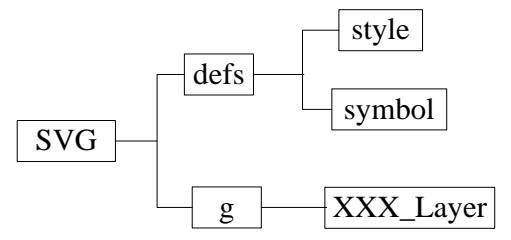

Fig. 1. The file structure of SVG format.

Among them, xxx_Layer includes Breaker_Layer, Disconnector_Layer, GroundDisconnector_Layer, Text_Layer, Bus_Layer, Load_Layer, Reactor_Layer, Transformer3_Layer, Transformer2_Layer, Generator_Layer, Link_Layer and Other_Layer.

\section{B. File Structure of $G$ Language Format}

$\mathrm{G}$ language format file adopts the three-level reference mode of power equipment graphics object - > bay template - > power equipment element. Element consists of basic graphics elements, such as line, rectangle, eclipse, circle, arc, elliptic arc, broken line, polygon, static text, pin and so on. Bay template consists of element and basic graphics element. Power equipment graphics object consists of bay, element and basic graphics element. The $\mathrm{G}$ language file structure of power equipment element, bay template and power equipment graphics object is as shown in figure 2. SymbolType represents the type of equipment element. Element represents equipment element. Bay represents bay. The entire monitoring graphics of power system are described through hierarchical reference.

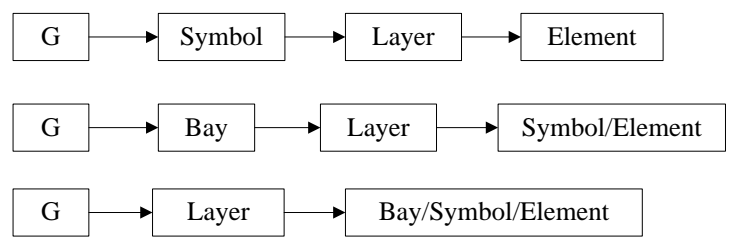

Fig. 2. The G language file structure of element, bay template and graphics.

Element is comprised of line, rect, circle, pin, polygon, polyline, ellipsearc and text. Symbol is comprised of CBreaker, Disconnector, GroundDisconnector, CT, PT, Generator, Arrester, Ascoil, Capacitor, Reactor, Terminal, ACLineEnd, ACLine, Transformer3, Transformer2, Tex, DText and ConnectLine. Bay is comprised of dbo, sbo, dbus, dcb, threequarters and two-part.

\section{DESIGN AND IMPLEMENTATION OF PROGRAM}

The input of program is SVG file, and the output are hierarchical and classified metafile set of $\mathrm{G}$ language, graphics files of $\mathrm{G}$ language and point tables for signal retransmission. The graphics transformation ideas are as below. Firstly, SVG file is parsed. Secondly, the node of defs/symbol is analyzed, and the hierarchical and classified metafile set of $G$ language are generated according to the mode of element type and state fusion. Thirdly, the graphics files of $\mathrm{G}$ language are generated 
according to the corresponding relationship of power graphics elements. At the same time, the point tables for signal retransmission are generated as the data source of graphics refreshing for remote browsing [5].

The whole program is divided into three modules. The SVGParser module for parsing SVG file is responsible for parsing the element and graphics node of SVG. The GElementConverter module for generating $G$ element file is responsible for generating the hierarchical and classified metafile set of $\mathrm{G}$ language. The GDisplayConverter module for generating $\mathrm{G}$ graphics file is responsible for generating the graphics file of $\mathrm{G}$ language and corresponding point table for signal retransmission. Among them, SVGParser is split into three sub modules. The sub module of Decompose is responsible for decomposing the complex attribution into some unit attributions and incorporating into the sub module of element and equipment graphics layer. The sub module of Element is responsible for parsing the node of element and generating basic graphics elements set of all elements. The sub module of Layer is responsible for parsing the node of graphics layer and generating equipment graphics elements set of all layers. The overall design framework is as shown in figure 3 .

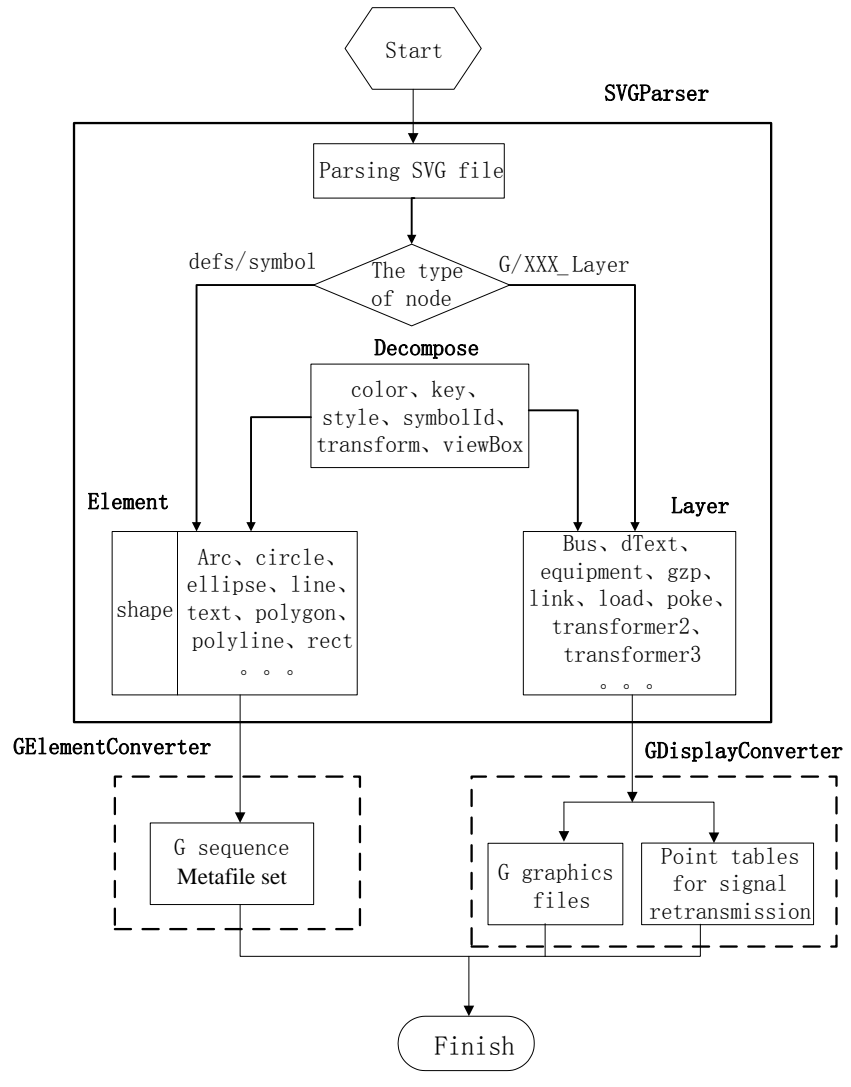

Fig. 3. The overall design framwork diagram.

\section{ENGINEERING APPLICATION}

The graphics conversion method proposed in this paper has been applied in some demonstration projects of remote browsing for ultra high voltage substations, such as $500 \mathrm{kV}$ jiupan substation in Chongqing, 500kV miaoxi substation in Zhejiang, $750 \mathrm{kV}$ qianxian substation in Xi'an, $500 \mathrm{kV}$ ciyun substation in Hebei, $500 \mathrm{kV}$ banqiao substation in Tianjin and so on. The SVG monitoring graphics in substation can generate $\mathrm{CIM} / \mathrm{G}$ graphics file through vector graphics file conversion. Then, the CIM/G graphics file can be sent to the dispatching terminal by the transmission way of file content data block in DL476 protocol. At last, after the graphics files are reduced and displayed by the receiving module of the front of dispatching and graphics system, the graphics and data of the dispatching master browsing are the same as the graphics and data of substation. The method of graphics conversion is verified by the consistency, reaching the functional requirement of remote browsing.

\section{CONCLUSION}

The conversion method from SVG graphics for substation to $\mathrm{CIM} / \mathrm{G}$ graphics for intelligent dispatching leaps over two vector graphics standards for power system. The communication bridge is erected from SVG for substation to $\mathrm{CIM} / \mathrm{G}$ for the dispatching master, realizing seamless docking of two vector graphics. The graphics normalized working time of remote browsing for substation can be shortened greatly. The field engineers can be liberated from the jobs of tedious drawing, linking library and checking point. The efficiency of field consistent debugging can be improved greatly. The engineering pressure can be reduced greatly. The human, material and financial investment for the substation construction can be saved. Proved by engineering practice, the converted $\mathrm{G}$ language format graphics files meet the functional requirements for remote browsing completely, and under the requirements for the large maintenance system, remote operation and maintenance for substation equipment can be realized, thereby greatly improving the level of unattended substation.

\section{ACKNOWLEDGMENT}

This work was financially supported by the Science and Technology Project of State Grid Corporation of China, the name of which is "The Research and Application of Key Technology for Equipment Monitoring Business Under the Mode of Large Operation" (Project No. DZ71-14-039).

\section{REFERENCES}

[1] HOU Yu, LI Suyou. SVG technology and its application based on XML[J]. Application Research of Computers, 2002(5): 136-138.

[2] GUO Zhenhui, Qu Xiaohong, WANG Hao. Using SVG in substation graphics[J]. Power System Technology, 2006 (30): 446-450.

[3] Q/GDW 624-2011. Graphic description specification for electric power system[S].

[4] LI Yongjun, JI Wenli, MA Guangsi. Using DOM4J to parse XML document[J]. Computer Applications, 2001 (8): 103-105.

[5] GAO Yanmin, JIANG Xiangang, WU Xiaolin. The design and implementation of the conversion from the customized vector graphics to SVG format[J]. Electric Power Information Technology, 2005(4): 6063. 